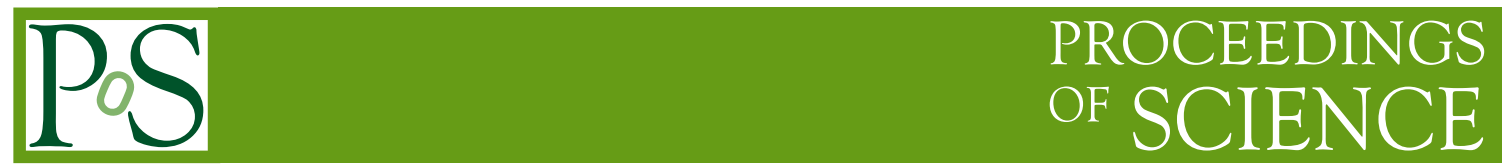

\title{
LHCb Detector Commissioning: Results with Cosmics and First Beam
}

\author{
Malcolm John* \\ University of Oxford \\ E-mail: malcolm.john@physics.ox.ac.uk
}

With high-energy collisions at the LHC imminent, this contribution describes the final commissioning of $\mathrm{LHCb}$ detector with comics and non-circulating beam. The status of each major subdetector is reported, with particular attention paid to recent highlights.

12th International Conference on B-Physics at Hadron Machines - BEAUTY 2009 September 07 - 112009

Heidelberg, Germany

${ }^{*}$ On behalf of the LHCb collaboration 


\section{Physics motivation in a nutshell}

Over the last decade, several experiments have been very successful in demonstrating the effectiveness of the Standard Model in describing heavy quark phenomenology. Perhaps most notably, the measurements of $\mathrm{CP}$ violation in B-decays, across a wide range of decay modes, have been found to be entirely consistent with the single irreducible phase that is built-in to the CKM mechanism of quark-mixing.

With LHCb, the focus turns to using precision flavour physics as a laboratory in which searches for new-physics effects may be performed. By examining decays that have proven too rare or too difficult for prior experiment, $\mathrm{LHCb}$ aims to test the Standard Model (SM) in five broad areas:

- Measuring the size of $\mathrm{CP}$-violation in $\mathrm{B}_{\mathrm{s}}^{0}$-mixing. Recent results from the Tevatron suggest a large deviation from the SM's small expectation. [1] LHCb is equipped with superior vertexing and a larger boost compared to previous experiments and it is expected that $\mathrm{LHCb}$ will make a competitive measurement with the 2010 data. [2]

- The branching fraction and kinematic properties of several di-muon B-decays have been identified as having sensitivity to new physics in loop diagrams. The most promising of these modes are $\mathrm{B}_{\mathrm{s}}^{0} \rightarrow \mu^{+} \mu^{-}[3]$ and $\mathrm{B}_{\mathrm{d}}^{0} \rightarrow \mathrm{K}^{*} \mu^{+} \mu^{-}$. [4]

- Precision measurements of the CKM parameter $\gamma$ are needed to advance the verification of the SM. At LHCb measurements of $\gamma$ in both tree [5] and loop processes [6] will yield results independent of, and sensitive to, new-physics couplings.

- Sensitivity to anomolous right-handed currents can be tested for by measuring the polarisation of the photon in radiative B-decays decays like $\mathrm{B}_{\mathrm{s}}^{0} \rightarrow \phi \gamma$ and $\mathrm{B}_{\mathrm{d}}^{0} \rightarrow \mathrm{K}^{*} \gamma$. [7]

- $\mathrm{LHCb}$ has a rich charm programme which includes a measurement of CP-violation in Dmixing and searches for rare muonic D decays. [8]

A detailed review of the $\mathrm{LHCb}$ physics programme can be found in [9].

\section{LHCb detector overview}

The LHCb detector has been conceived to take advantage of the huge $b \bar{b}$ cross-section expected in multi-TeV proton-proton collisions. A cross-section of $\sim 500 \mu \mathrm{b}$ is estimated at $\sqrt{s}=$ $14 \mathrm{TeV}$ with $\sim 230 \mu \mathrm{b}$ containing a b-hadron with $p_{\mathrm{T}}$ larger than $2 \mathrm{GeV} / c$.

The overall design of $\mathrm{LHCb}$ is that of a spectrometer with an instrumented dipole magnetic field placed in the forward region of the proton-proton collisions. Particle tracking is achieved using a mixture of straw-tube and silicon technologies and covers a pseudo-rapidity range of $1.9<\eta<4.9$. Even with this reduced angular coverage, LHCb can fully reconstruct approximately $40 \%$ of the B-mesons decaying in the forward hemisphere. The detector is described in detail in [10]. 
To perform B-physics at the LHC, a specialist detector and a highly selective trigger is required to identify only the most interesting decays. Detection of the characteristic properties of heavyquark hadrons: large $p_{\mathrm{T}}$ and a displaced vertex, are the foundation of the design of $\mathrm{LHCb}$, see Fig. 1. A fast, hardware trigger (L0) is designed to select high- $p_{\mathrm{T}}$ objects using the calorimeter and muon systems. The L0 thresholds are set such that whole detector is then read-out into a software trigger (HLT) at a rate of $\sim 1 \mathrm{MHz}$. The first task of the HLT looks to confirm the L0-object before using the vertex detector to search for a displaced vertex. The final part of the trigger uses the full spectrometer information and is tuned to accept decay modes of interest at a rate of $2 \mathrm{kHz}$.

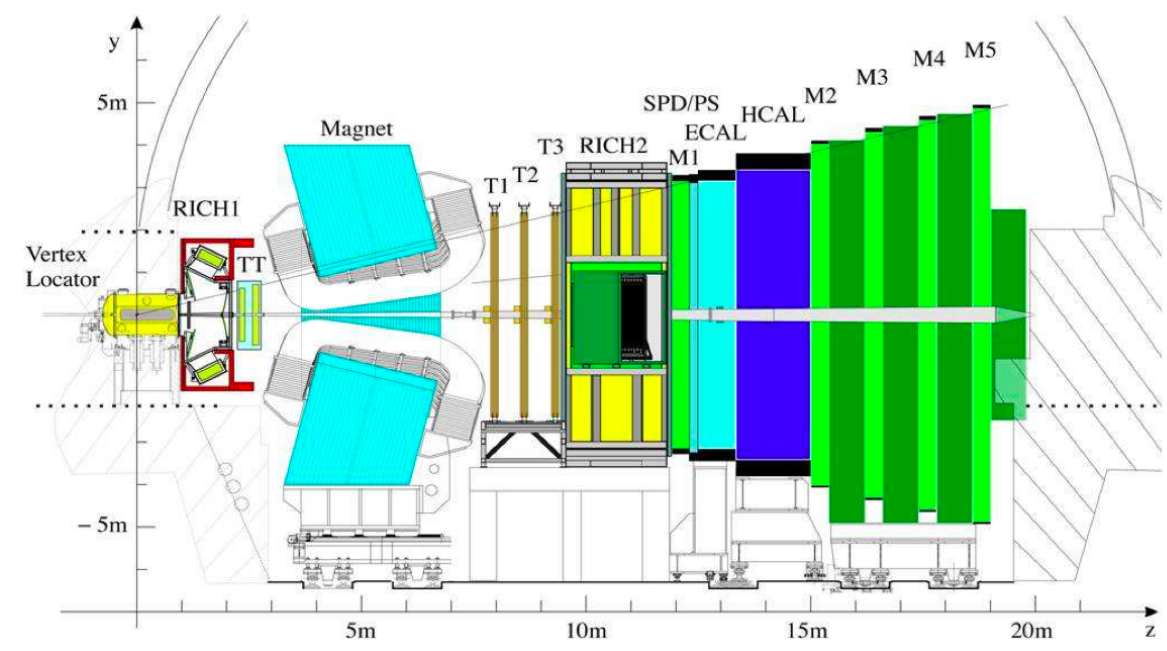

Figure 1: The layout of $\mathrm{LHCb}$ as viewed from inside the LHC ring. The dipole magnet is the dominant feature, lying between the tracking stations: TT and T1,2,3. A muon filter (M1-5) lies behind the calorimeters (ECAL \& HCAL) and dedicated particle identification is provided by two RICH counters. The interaction point is at $\mathrm{z}=0$, in the centre of the silicon vertex locator.

During 2008 and 2009 a combination of strategies has been used to commission LHCb. Calibration pulses are used to check control and data cables as well as the various databases and readout mappings. After some debugging and masking of dead or noisy channels, over $99 \%$ of the detector is seen to be functioning correctly. Recently, the entire DAQ chain has been exercised, demonstrating the design readout rate of $1 \mathrm{MHz}$ and a $2 \mathrm{kHz}$ write-to-tape frequency.

\section{Commissioning of the large sub-detectors with cosmic rays}

Due to its horizontal geometry, $\mathrm{LHCb}$ is not well suited to recording cosmic rays. Nevertheless, by relaxing the hardware trigger criteria, a rate of about $1 \mathrm{~Hz}$ has been achieved. For this, the thresholds on $p_{\mathrm{T}}$ and $E_{\mathrm{T}}$ are reduced and a coincidence of just two of five muon stations is required. Many runs have been taken with these conditions over 18 months, collecting several million useful cosmic events.

The cosmic-ray sample permits the large detectors of $\mathrm{LHCb}$ - calorimeters, muon system and the straw tracker - to perform time and spatial alignment exercises. An example of this is shown 
in Fig. 2 which show the response of four muon stations with respect to a trigger. In this case, the trigger is provided by by the hadron calorimeter and is adjusted for tracks traveling forward from M1 to M5. The obtained time resolutions of 4 ns are in agreement with expectations.

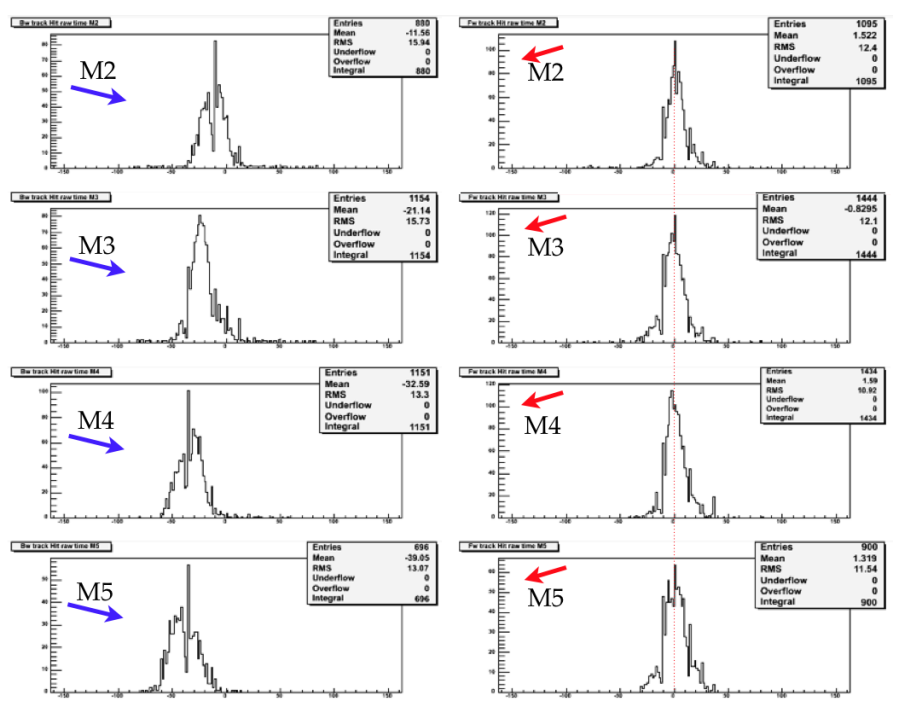

Figure 2: Time resolution distributions for four muon stations from minimum-ionising cosmic rays traveling through $\mathrm{LHCb}$. The muon system response is tuned for the forward tracks [right]. The equivalent distributions for tracks traveling backward through $\mathrm{LHCb}$ are included for comparison [left].

For the straw-tube outer tracker (OT) an alignment procedure is applied to the 216 detector modules using the fully cosmic-ray dataset. A typical cosmic ray used in the calibration is visualised in Fig. 3 where the high-energy track is seen depositing energy in all of the large detectors. Fig. 4 shows the result of the OT alignment exercise. After calibrating the drift-times of the straws, a hit resolution of $88 \pm 6 \mu \mathrm{m}$ has been achieved.

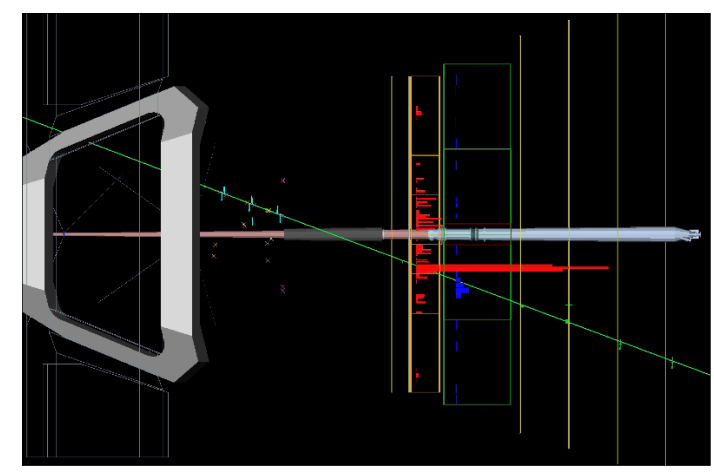

Figure 3: An example cosmic-ray event that enters from the left; it passes though the OT, then leaves an energy deposit in the ECAL and HCAL before passing though the muon system.

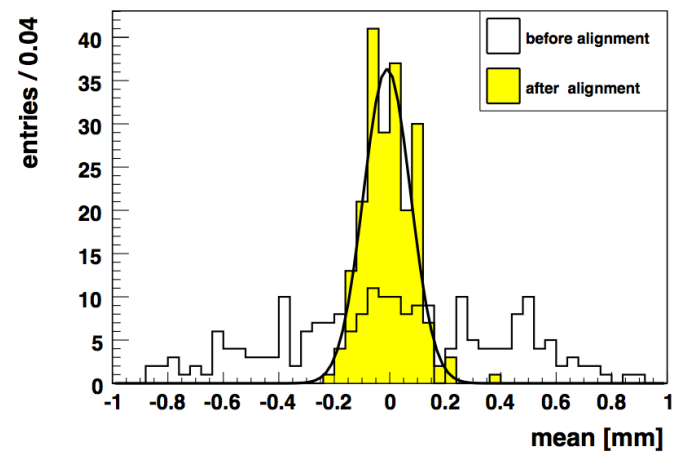

Figure 4: Hit resolutions in the OT before and after alignment. The hit resolution after alignment is measured to be $88 \pm 6 \mu \mathrm{m}$. 
For the small-area silicon detectors is its not practical to collect alignment data from cosmic tracks and an alternative calibration method has been employed.

\section{Commissioning the silicon detectors with non-circulating beam}

During the routine commissioning of the LHC, beam sychronisation tests were performed where single bunches of protons are delivered at 48 second intervals down the transfer-line that connects the SPS with the LHC. For several days in 2008 and 2009, These $450 \mathrm{GeV}$ particles were then incident on the TED (Transfer-line External Dump) which is located $340 \mathrm{~m}$ downstream of LHCb. The resulting showers of secondary particles passed though the LHCb cavern with a flux of approximately 10 particles $/ \mathrm{cm}^{2}$.

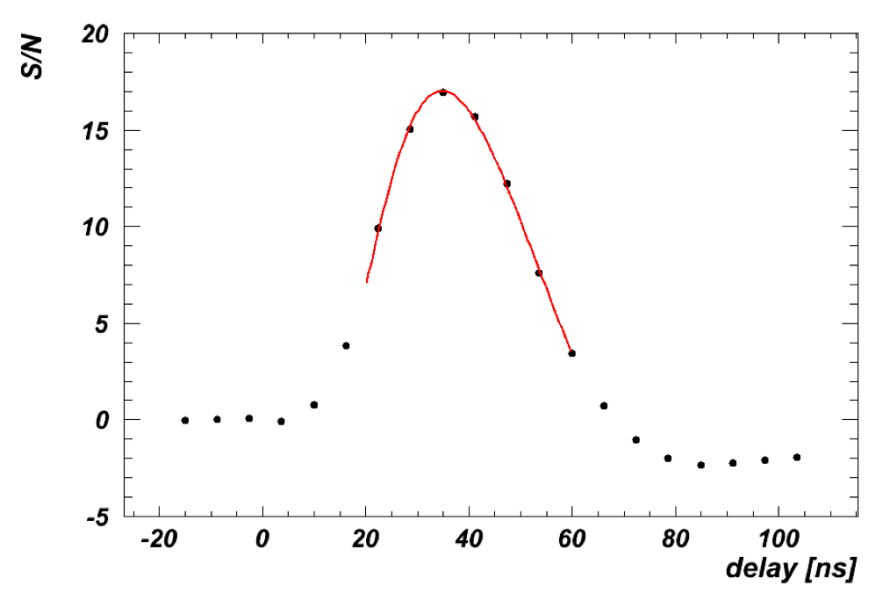

Figure 5: The signal-to-noise ratio for an IT module as a function of delay from the trigger signal.

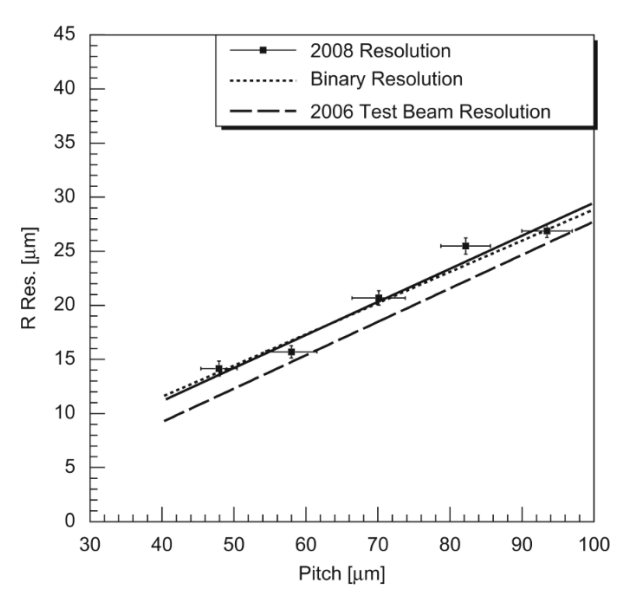

Figure 6: Hit resolution of VELO Rsensors as a function of strip pitch.

With these charged particles various aspects of the VELO and silicon tracker data-acquisitions systems were tested for the first time. Timing studies were performed where fine-delay settings in the front-end chips were systematically varied in order to identify the optimal timing. An example of the response of an inner tracker (IT) module as a function of fine-delay is shown in Fig. 5.

The vertex-locator (VELO) consists of 84 planes of silicon microstrip sensors; half have strips arranged in a radial geometry, the other 42 define the azimuthal angle. On each sensor, the strip pitch varies from $40 \mu \mathrm{m}$ at the inner edge to $100 \mu \mathrm{m}$ at largest distance from the beam-line. An alignment procedure has been completed using the tracks from the TED and the deduced displacements and rotations are compared with the result of a prior optical survey. The sensors were found to be displaced by less than $10 \mu \mathrm{m}$ with respect to their surveyed positions.

The hit resolutions after alignment are shown in Fig. 6 for VELO sensors with radial strips. The result show very good agreement with a binary performance - which is expected for the TED dataset in which over $90 \%$ of clusters consist of just one hit-strip. It should be noted that for collision data, the resolution of the VELO sensors will be somewhat improved due to the added 
precision afforded by the charge-sharing between adjacent strips. In $14 \mathrm{TeV}$ pp-collisions, tracks are expected to traverse the VELO sensors with an average incident angle of $10^{\circ}$.

\section{First rings in the LHCb RICH}

LHCb benefits from dedicated particle identification courtesy of two ring-imaging Cherenkov (RICH) counters. Due to the use of three radiators, the LHCb RICH system can resolve kinematically similar hadronic final states over a momentum range from 2 to $\sim 100 \mathrm{GeV} / c$. The upstream RICH1 uses $\mathrm{C}_{4} \mathrm{~F}_{10}$ and aerogel to cover the lower momentum range and RICH2, downstream of the spectrometer magnet, contains $\mathrm{CF}_{4}$ to provide particle ID for higher momentum tracks.

The TED data has been used for time alignment with a trigger supplied by the calorimeter. However, these tracks do not produce rings in the RICH as the charged particles must be traveling forward in $\mathrm{LHCb}$ for the rich-imaging optics to work correctly. More specifically, to form nearcircular rings, the charged-tracks should appear to originate from near the interaction point.

To complete the commissioning and see rings, two large scintillators were placed around RICH1; one in front of the VELO tank and one immediately upstream of the RICH1 vessel. Their coincidence provided an auxiliary trigger to the data acquisition and candidate Cherenkov rings were searched for in the data.

Fig. 7 displays a selection of the first Cherenkov rings seen in the LHCb RICH. In each example the smaller ring originated in a gas radiator and their size is consistent with expectation for a $\mathrm{CO}_{2}$ radiator $^{1}$. Furthermore, many of these gas rings are encircled with an arc of photons which is assumed to be part of the accompanying Cherenkov ring from the aerogel radiator. Finally, the quietness of these displays, which each represent over $10^{5}$ readout channels gives some indication of the excellent noise performance of the pixel-chip hybrid photo-detectors and their encased front-end electronics. [11]

\section{Conclusion}

$\mathrm{LHCb}$ is in an excellent state of preparation prior to the first collisions. All sub-detectors have demonstrated greater than $99 \%$ functional and all have been exercised with charged particles from either cosmics or from secondary particles resulting from accelerator commissioning. The full detector has also been read out at a maximum hardware trigger rate of $1 \mathrm{MHz}$ and is fully operational for the first physics run in 2010.

\footnotetext{
${ }^{1} \mathrm{RICH} 1$ contained with $\mathrm{CO}_{2}$ at during this time. The volume has since been filled with the design radiator, $\mathrm{C}_{4} \mathrm{~F}_{10}$ in preparation for physics data-taking.
} 

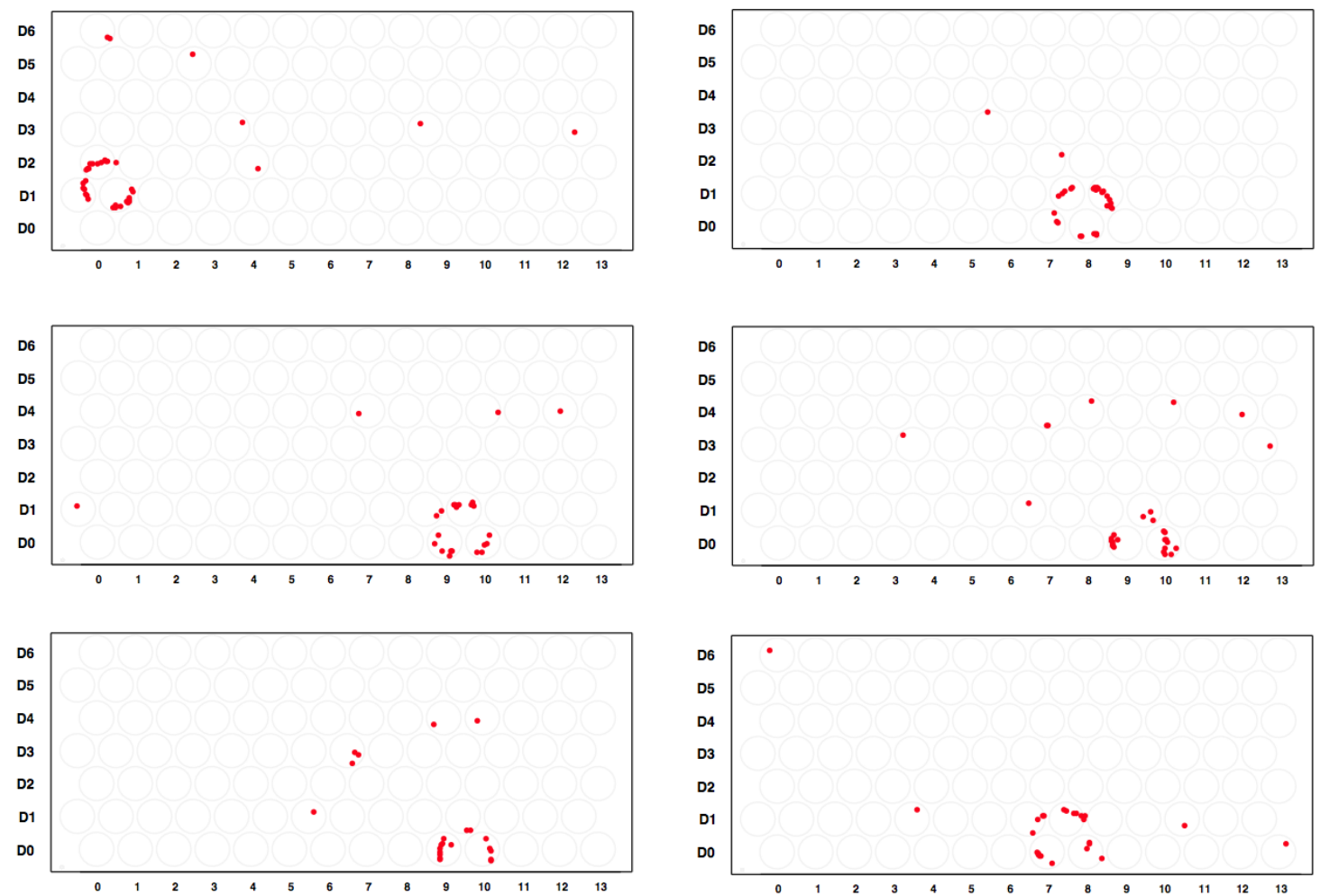

Figure 7: A selection of the first Cherenkov rings seem by the fully commissioned LHCb RICH1 detector. Rings from the gas radiator a clearly identifiable. Most exhibit accompanying larger ring patterns consistent with being Cherenkov photons from the aerogel radiator.

\section{References}

[1] K. Gibson, "CP-violation in $\mathrm{B}_{\mathrm{s}}^{0} \rightarrow \mathrm{J} / \psi \phi$ at the Tevatron", these proceedings

[2] O. Leroy, " $\mathrm{B}_{\mathrm{s}}^{0} \rightarrow \mathrm{J} / \psi \phi$ and $\mathrm{B}_{\mathrm{s}}^{0} \rightarrow \phi \phi$ at LHCb", these proceedings

[3] D. Martinez Santos, " $\mathrm{B}_{\mathrm{s}}^{0} \rightarrow \mu^{+} \mu^{-}$at LHCb", these proceedings

[4] W. Reece, "B $\rightarrow \mathrm{K}^{*} \mu^{+} \mu^{-}$at LHCb", these proceedings

[5] S. Ricciardi, "Tree-level $\gamma$ determination at LHCb", these proceedings

[6] L. Carson, "Charmless B-decays at LHCb", these proceedings

[7] I. Belyaev, "LHCb radiative decays", these proceedings

[8] J. Magnin, "Charm physics at LHCb", these proceedings

[9] LHCb Collaboration, "Roadmap for selected key measurements of LHCb", arXiv:0912.4179, 2009.

[10] LHCb Collaboration, "The LHCb Detector at the LHC", JINST 3 S08005.

[11] M. Moritz et al., IEEE Trans. Nucl. Sci. NS-51 (3) (2004), p. 1060. 